EOMmun: Communication et organisation

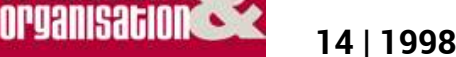

Les formations à la communication

\title{
Identité européenne et communication
}

Jean-Pierre Callégari

\section{OpenEdition}

Journals

Édition électronique

URL : http://journals.openedition.org/communicationorganisation/2114

DOI : 10.4000/communicationorganisation.2114

ISSN : 1775-3546

Éditeur

Presses universitaires de Bordeaux

Édition imprimée

Date de publication : 1 novembre 1998

ISSN : 1168-5549

\section{Référence électronique}

Jean-Pierre Callégari, «Identité européenne et communication », Communication et organisation [En ligne], 14 | 1998, mis en ligne le 26 mars 2012, consulté le 19 avril 2019. URL : http://

journals.openedition.org/communicationorganisation/2114; DOI : 10.4000/

communicationorganisation. 2114

Ce document a été généré automatiquement le 19 avril 2019

(c) Presses universitaires de Bordeaux 


\title{
Identité européenne et communication
}

\author{
Jean-Pierre Callégari
}

\section{NOTE DE L'ÉDITEUR}

Nous tenons particulièrement à exprimer notre gratitude à Monsieur J.P. Malivoir.

Directeur a la DG X (Direction Générale Information, Communication, Culture.

Audiovisuel de la Commission Européenne), pour son amabilité et l'entretien clarifiant qu'il a bien voulu nous accorder, pour les documents qu'il a bien voulu nous adresser et qui explicitent la stratégie de communication conduite par l'Union Européenne.

Devenue l'Union Européenne avec le traité de Maastricht après avoir été C.E.E. pour l'Économie, U.E.O. pour la Défense, Communauté Européenne suite à l'Acte Unique en 1986, l'Europe de l'Ouest est sans doute une organisation originale d'une région du monde, certainement pas un pays ni une nation. Elle ne doit pas moins affirmer une identité fondement de son existence, face au reste du monde, d'abord au regard des quelques 370 millions de personnes qui habitent les six puis neuf, douze, quinze entités... qui la composent, sans jamais apparaître une menace pour les unes ni les autres.

2 Pour exister à la face du monde en dépit de ses disparités, l'Union Européenne doit donc instiller la notion de territoire, créer un espace intermédiaire entre l'espace-monde et l'espace Etat-Nation où les régionalismes seraient préservés, donc afficher une spécificité qui reflète sa diversité spatioculturo-économique, tout en ménageant les microcultures régionales qui font sa richesse; en fait générer une identité qui se construit toujours dans un relationnel avec l'autre.

3 La communication apparaît le meilleur moyen de générer cette prise de conscience et de gérer une démarche recouvrant une logique segmentation/globalisation, une dialectique qui renvoie au modèle intégration/différenciation souvent associé au développement organisationnel dans les entreprises; les contextes ne sont pas sans analogies. 
4 Le Conseil Européen de Fontainebleau a donc souhaité promouvoir une sorte d'espace intermédiaire, son image auprès des citoyens de l'Union et dans le monde, en fait une identité, donc une culture avec ses valeurs associée à un territoire, l'une n'allant pas sans l'autre.

5 Il a chargé la DG X, d'impulser la démarche de communication idoine, à même d'accompagner les évolutions, la maturation de l'identité. En réalité, la DG X a plus un rôle de coordination dans ce domaine puisque chaque Direction Générale intègre maintenant dans ses programmes un volet communication, et que celle-ci reste l'apanage des États de l'Union. Cette situation implique effectivement une dialectique, explique le choix de la stratégie de communication de la Commission qui s'inscrit davantage dans une double perspective, fonctionnaliste et interprétative, mais doit aussi réussir une synthèse entre celle-ci et une nécessaire communication de proximité. L'Euro pourrait en être le filigrane, de par son nom et son usage dans la vie quotidienne des européens.

\section{Une communication institutionnelle symboliquement fédératrice sur l'Europe}

6 Partant de ce qui la définit et de son objectif, fédérer les peuples sur l'Europe en interne et face au reste du monde, la DG X a en fait développé une stratégie fondée sur la persuasion et la médiation, sur une idée louable mais difficile, visant à concilier la communication qui parle au cœur et l'information qui parle à la tête, d'où les différents volets et supports de sa stratégie.

7 Tant pour l'espace-europe que pour l'espace-monde, elle a développé des symboles, porteurs de valeurs fédératrices qui dépassent les états et leur histoire: le rituel du drapeau aux douze étoiles symbolisant la plénitude et la perfection, de "l'Hymne à la Joie » pour emblème musical, du 9 mai « Journée de l'Europe » pour célébrer les objectifs de paix et de solidarité, du mécénat d'événements importants, notamment sportifs pour éviter au mieux que la communication corporate persuasive puisse s'assimiler à la propagande ou la promotion d'une marque commerciale.

8 Elle entend faire de l'Euro un autre symbole choisi au terme d'un concours et d'une étude de marché sur une tranche d'âge allant de 18 à 75 ans, qui combine modernisme et classicisme, veut exclure toute interprétation artistique mais signifier par ses lignes, ses angles et couleurs, la stabilité espérée par les populations et que veut apporter l'Union.

9 Faute de pouvoir procéder d'une histoire européenne paisible, elle est en effet obligée de recourir aux symboles pour leur valeur d'image signifiante au sens sémiologique, aussi parce qu'elle parie sur une histoire future néanmoins imprégnée d'une culture passée et intemporelle ; autrement dit paraphrasant à peine R. Sainsaulieu, pour mieux permettre aux «communautés traditionnelles» de se dissoudre et s'installer dans un avenir maitrisé par "une sorte de collégialité contingente». Gardons en effet à l'esprit que la première modalité collective de l'identité renvoie à l'appartenance, la seconde et la troisième à la réalisation de soi au travers d'une action construite, la quatrième à la résistance à toute forme de domination perçue comme imposée.

10 Aussi, la DG X joue-t-elle également la proximité et une certaine modestie dans une communication informative, en installant des Bureaux de Représentation de la Commission dans chaque pays, des Centres de Documentation et de Recherche dans les Régions, les Euro-Info-Centres pour les entreprises ou Euro-guichets devenant parfois 
Centres de Correspondance pour l'espace-monde. Sans doute est-ce dans le même esprit qu'elle soutient le petit fascicule au titre significatif jusque dans sa graphie «demain l'euro/ensemble apprenons l'euro", qui veut familiariser à l'utilisation au quotidien d'une monnaie dont les pièces auront une face Europe commune et une face spécifique à chaque pays.

11 Ainsi l'Euro devient-il vecteur de communication signifiant des valeurs à promouvoir, figurant du double espace, Europe et État-Nation, auquel les citoyens peuvent adhérer sans craindre de se renier ni de déstructurer leur identité ${ }^{1}$, puisque les deux faces participent du même tout et le constituent.

\section{Une prudente communication institutionnelle de proximité}

12 Échaudée par la désillusion née de la démarche de communication pour le Marché Unique, la DG X est en effet attentive à transformer un "consensus passif » en "participation active", en résumant l'évolution de sa stratégie par trois mots clefs figurant sur la couverture du recueil de 1994 «Information, Communication, Transparence ».

Vouloir expliquer, écouter et ne rien cacher, évaluer l'impact de tous les supports, jouer les réseaux internes et externes, multiplier les enquêtes et eurobaromètres, réunir deux fois par an un conseil consultatif des usagers composé de représentants de la presse et autres catégories socioprofessionnelles s'ajoutant aux universitaires, cadres d'entreprises plutôt importantes, membres des relais institutionnels, manifeste un progrès indéniable néanmoins en deçà des attentes d'un grand public. Celui-ci n'appartient pas forcément aux quelques $15 \%$ de la population maitrisant l'anglais, parle l'une des 11 langues voire l'un des 59 dialectes, mais ne comprend pas toutes les brochures aux titres pourtant souvent prometteurs, diffusées par les euro-guichets, parce que les traductions ne correspondent pas tout à fait à sa culture et lui insufflent le sentiment, fut-ce à tort, qu'on tente de lui imposer de loin un idiome qui n'est pas le sien. Autrement dit un public dont les peurs se sont déplacées, qui craint surtout pour son identité et un univers sécurisant dans lequel il se reconnaît, perçoit plus la précarité de son emploi comme une conséquence des critères de convergences inspirés du Traité de Maastricht. Le sociologue o. Galland rappelle dans l'ordre que la famille et le travail, avec le relationnel et le respect de la vie personnelle restent des "valeurs indétrônables". Même s'il les réintègre dans le concept de culture et souligne celui du jugement, L. Thévenot, généralise en regroupant les valeurs de progrès, égalité, mérite d'une part, la fidélité, l'amitié, la convivialité, la communauté, l'ancrage dans l'histoire et le passé d'autre part. Quant à J. Lecomte, il ne se démarque pas davantage en distinguant les valeurs personnelles, instrumentales, morales et de compétences.

14 La revendication d'accomplissement et d'autonomie dans le changement, la recherche de " satisfaction autocentrée » ajoutées à la notion de valeur comme fondement de l'identité et critère d'adhésion font mieux comprendre le mode de communication à impulser, la prudence et le souci de la DGX d'informer dans la transparence et de veiller à l'éthique dans la communication pour réussir à concilier le sentiment d'appartenance qui relèverait davantage de l'échelon local ou régional, le sentiment d'identité surtout national, la perception de l'enjeu économique plutôt global ou européen. 
15 C'est pourquoi, presque exclusivement à destination de "l'intérieur », elle a privilégié une communication écrite, informative, didactique, avec toutes les publications à caractère documentaire pour expliquer les actions; mais le résultat est mitigé vu le foisonnement des informations parfois rédigées dans un «eurojargon", souvent prioritairement en anglais, pour les appels d'offres notamment.

C'est pourquoi dans son souci de réconcilier avec et sur l'Europe, de provoquer l'adhésion en interne pour mieux l'affirmer à et par l'externe, la communication impulsée s'appuie sur des valeurs au bien fondé indiscutable, recherche la transparence pour ménager des populations perplexes sinon méfiantes, la proximité pour rapprocher les institutions du public-cible, en multipliant les brochures disponibles par un réseau d'euro-guichets, et censées répondre aux questions que se poseraient les particuliers ${ }^{2}$, en déléguant des fonctionnaires courtois et formés à la communication dans toutes sortes de réunions pour mieux expliquer la politique de la Communauté ; en cherchant à mieux connaître les attentes des européens sur l'Union en général, l'Euro en particulier qui recueille $54 \%$ d'opinions favorables dans l'enquête réalisée par le C.R.E.P. en juin 1997.

\section{Les manques identitaires d'une cohérence stratégique ratée}

C'est pourquoi la Commission a aussi recherché la cohérence en lançant par exemple le programme MEDIA pour relever le pari de la télévision avancée, en faisant des N.T.I.C. (Nouvelles Technologies de l'Information et de la Communication) et des autoroutes de l'information, un axe majeur du dernier Programme Cadre de Recherche et Développement.

Mais est-il dans le même temps toujours bien cohérent, peut-être par réflexe supranational, de flatter des identités régionales, méditerranéenne ou atlantique, et de provoquer des crispations nationales alors que la communication reste l'apanage des États et que les citoyens européens comptent d'abord sur leur État?

De même il est judicieux de penser la communication graphique pour visualiser une identité, dépasser les freins linguistiques et atteindre sa cible, car les signes iconiques contenus sont « des objets autant que des sujets dans les stratégies d'influence » ${ }^{3}$. C'est en outre un moyen de dépasser certains freins sans dispenser de l'écrit. Les meilleurs textes et les meilleures traductions perdent de leur impact si elles n'intègrent pas les niveaux de lecture, les différences culturelles; ainsi les français apprécieraient le caractère pédagogique ; les non-français privilégieraient l'appartenance au groupe ; tous la mise en valeur des équipes. Les entreprises multinationales engagées dans une communication externe fédératrice l'expérimentent tous les jours. D'où l'intérêt de procéder de l'identité, dont le caractère dynamique et multimensionnel correspond à la revendication fondamentale des citoyens européens, pour engager une démarche de communication cohérente. L'identité européenne doit participer de l'identité nationale, comme l'espace état-nation de l'espace-europe.

Faire le choix d'une stratégie de communication qui permet de croiser la perspective fonctionnaliste et les processus de persuasion paraît a priori judicieux dans la mesure où on peut recourir à un numéro vert et aux supports écrits, graphiques, sonores, audiovisuels insérés dans un système résiliaire pour assurer le martèlement souhaitable et promouvoir une certaine proximité ; dans la mesure aussi où il est possible, pour 
réussir à influencer des attitudes et insuffler des idées nouvelles, d'associer le statut ou la légitimité de la source d'information à l'expertise de l'émetteur, à faire apparaître dans ce mode minoritaire «disputant» dont parle S. Moscovici, donc sous réserve de rétablir ainsi un niveau d'équilibre entre émetteurs et récepteurs, partenaires interactivement à même d'induire le principe d' " unir ensemble » dans l'acte de communiquer.

Cette approche fait mieux correspondre la perspective fonctionnaliste à une volonté fédératrice propice à la gestion des relations, autorise davantage la perspective intégratrice qui ménage une place à la transaction, à une dynamique favorisant pour le récepteur surtout, appropriation et création d'une identité susceptible de devenir européenne, qui commence généralement par être personnelle. Cependant, même renouvelée dans le bon sens, favorable à l'objectif visé donc, cette approche ne révèle pas moins la difficulté, l'ambiguïté de cette stratégie de communication corporate, une certaine incohérence alors qu'elle est en fait supposée le contraire.

Ainsi en arrive-t-on au défaut fondamental de ce type de stratégie. La communication institutionnelle est tentante parce qu'elle propose un partenariat contractuel, dynamique qui n'est pas sans rappeler le processus identitaire correspondant. Plus fondamentalement, pour réussir encore faut-il s'appuyer sur une «doxa» chère à $P$. Bourdieu; or c'est justement ce qu'elle entend élaborer. Pour obtenir des populations des changements de comportements profonds et durables ceux-ci doivent aussi s'accompagner de mesures techniques, surtout économiques; rien de moins facile et convaincant après le traité de Maastricht qui officialisa justement l'Euro et le Comité européen des régions. Enfin il n'est pas non plus aisé de réconcilier avec et sur l'Europe sans jouer sur la peur, alors que les controverses issues du Traité de Maastricht ont révélé dits et non-dits, craintes et replis ou résurgences de revendications, locales, régionales ou nationales. Cela est encore moins facile, avec un mode de communication principalement soucieux de transmettre des messages et modifier des attitudes mentales à partir d'une vision instrumentale des "phénomènes psychologiques de la persuasion » selon l'expression de J.N. Kapferer.

\section{Identité et Espace ou les ressorts et finalités d'une communication interculturelle cohérente}

23 S'il est en effet certain qu'après d'autres vecteurs, les Nouvelles Technologies d'Information et de Communication (N.T.I.C.) peuvent jouer un rôle déterminant, faut-il croire pour autant qu'ils vont fonder seuls, cet «espace interactif et mouvant... (où) les nouveaux moyens de création et de communication pourraient renouveler... les formes du lien social dans le sens d'une plus grande fraternité... autour de l'apprentissage réciproque, de la synergie des compétences, de l'imagination et de l'intelligence collective? »4. En regard de notre propos, l'intérêt du livre de Pierre Levy paraît être de replacer les N.T.I.C. dans une diachronie, de rappeler implicitement que, dans une communauté où «deux personnes disposées de part et d'autres d'une frontière sont (souvent) plus loin l'une de l'autre que des gens appartenant au même pays, même si le rapport est inverse dans l'espace de la géographie physique $»^{5}$. Indépendamment du caractère discutable de cette remarque et s'il est bon de créer une société de communication technologique, il faut aussi penser l'interaction des deux et commencer par créer une société de communication, soit "une unité structurale d'organisation et de transmission culturelle et sociale ${ }^{6}$. Dans telle région transfrontalière, la fibre optique n'a pas précédé les relations interpersonnelles. 
Les frontières peuvent représenter des Limites sécurisantes favorables à leur dépassement, constituer des zones de contacts plus que des barrières. Les programmes européens dits «Interreg ", le c conclusion semblable s'impose quant à la communication technologique instrumentalisée à laquelle il faut acclimater les citoyens qui ne le sont pas. De même il faut se garder de diaboliser la communication publicitaire lucrative sous le prétexte qu'elle serait plutôt inadaptée pour intéresser une majorité de gens à des domaines comme la santé, la sécurité, la solidarité, le social ou l'environnement.

Pour susciter une prise de conscience à la fois identitaire et spatiale, il ne convient pas a priori de jeter des anathèmes, caricaturer des modes de communication ou utiliser celleci pour faire de la désinformation, charger l'Union de tous les maux ou tenter d'accréditer des messages irréalistes. Il convient de chercher à construire une cohérence, un équilibre, une adéquation entre le contenu, la forme, le public, les situations, sans chercher à rendre ou faire percevoir l'émetteur omnipotent; revenir en fait par une démarche essentiellement cohérente à ce qui est le fondement de toute communication crédible laissant toute sa place à une interactivité vraie, où le verbal et le non verbal ouvriraient sur des espaces de liberté laissant à chacun des citoyens l'autonomie pour se déterminer et se positionner dynamiquement.

Il ne convient pas en effet d'essayer d'abattre les barrières culturelles par une communication de persuasion excessive ni de chercher à miner le terrain de l'identité culturelle, car la notion de sens inhérente à la communication découle du contexte, autrement dit de l'histoire, du mode de vie, de la culture qui fondent l'identité : l'image de soi qui distingue chacun de l'autre ; également l'image sociale que l'on construit à partir de la culture, également enracinée.

nous, espace et identité sont intimement liés et celle-ci suppose auss l'appartenance à un groupe. Celle-ci dépend également de la culture vue comme "l'ensemble plus ou moins fortement lié des significations acquises les plus persistantes et les plus partagées que les membres d'un groupe, de par leur affiliation à ce groupe, sont amenés à distribuer de façon prévalante sur les stimuli provenant de leur environnement et d'eux-mêmes, induisant vis à vis de ces stimuli des attitudes, des représentations, et des comportements communs valorisés, dont ils tendent à assurer la reproduction par des voies non génétiques ».

Puisque nous sommes assaillis de stimuli dans les situations rencontrées quotidiennement, ajoutons avec Cialdini que la communication doit clarifier et faciliter le "recours à nos stéréotypes, à nos règles empiriques pour classer les choses suivant une ou deux caractéristiques clés; ce qui nous permettra de réagir sainement et sereinement de façon machinale en présence de ces caractéristiques $»^{8}$. C'est tout l'intérêt de rappeler l'association valeur et jugement.

Vouloir réduire les peurs par une démarche de communication comme le souhaite la Commission Européenne est louable; encore faut-il commencer par réintégrer les identités culturelles qui sécurisent les individus et les aident à se repérer, puis penser la communication interculturelle qui « implique toujours des personnes..., qui véhiculent ou médiatisent les rapports entre cultures $»^{9}$. J.R. Ladmiral remarque à juste titre que «la communication interculturelle, c'est d'abord un problème de communication tout court, c'est-à-dire essentiellement un problème de communication verbale $»^{10}$, interpersonnelle avec ses dits et non-dits, dans laquelle le langage peut a priori représenter un frein ou un prétexte; témoin tel partenaire d'un programme communautaire voulant promouvoir en 
anglais les actions organisées, refusant la langue nationale de son pays à défaut d'utiliser celle de sa région, ce qui revient à mêler à rebours, territoire, culture et identité.

Cela souligne l'importance de comprendre les processus à l'œuvre dans une rencontre interculturelle, car le but ainsi que l'explique M. Abdallah Pretceille « $n$ 'est ni d'identifier autrui en l'enfermant dans un réseau de significations, ni d'établir une série de comparaison sur la base d'une échelle ethno-centrée... (mais de mettre l'accent) sur les rapports que le je (individuel ou collectif) entretient avec autrui ${ }^{11}$. Récemment invités à entendre leur directeur, les nombreux groupes de salariés d'une grande entreprise implantée internationalement avaient compris et partagé le message en dépit d'une méconnaissance d'une partie du vocabulaire, à cause d'exemples significatifs, de remarques concrètes appuyées sur quelques graphiques significatifs, surtout du comportement empreint de convivialité et d'empathie qui avait retenu leur attention, si l'on veut bien accorder foi aux résultats de l'enquête réalisée a posteriori.

31 Il n'est sans doute pas simple pour une institution coordinatrice d'une démarche de communication de réintégrer cet aspect, mais le favoriser aux réserves ou conditions évoquées plus haut revient en fait à resituer les fondements d'une communication persuasive ainsi rendue plus efficace, puisqu'on sait que si les médias de masse «peuvent stimuler le changement,... ce sont les communications interpersonnelles qui jouent le rôle crucial dans l'adoption ou le rejet définitif du comportement proposé „ ${ }^{12}$.

Promouvoir la construction d'une identité européenne dont chaque ressortissant serait porteur face au reste du monde sans que cette construction soit perçue comme une menace vis à vis de son identité et sa culture régionale ou nationale, comme une tentative de déculturation mais plutôt comme une aide à son enculturation et son acculturation, là paraît être l'objectif fondamental de la stratégie de communication à mener dans ce cas. Souvenons nous en effet que "la maturité de l'identité est... le dépassement des traces et problèmes laissés par l'histoire personnelle, individuelle ou groupale,... de conditionnement et... déformations acquises... par l'aptitude à intégrer des expériences nouvelles et à créer... une identité toujours en devenir $»^{13}$.

\section{Une communication de proximité vraie ou Pour dépasser les dualités identitaires et spatiales}

Réussir cela, requiert une aide, un apprentissage, une méthode, une logistique qui pourrait être l'Euro, mais commencerait par une accentuation significative des programmes européens, non fermés aux ramifications et opportunités, plus réactifs et souples dans le temps. Ils auraient vocation expérimentale par rapport à la stratégie de communication à exploiter.

Dans leur montage et leur réalisation, les programmes européens induisent une organisation et une logistique qui amènent les participants et bénéficiaires à se rencontrer, se découvrir sans se renier jusque dans leurs a priori, surmonter les difficultés une fois la relation de confiance installée, expérimenter la transaction en fait. Opportunités de communication interpersonnelles, ils représentent un moyen de faire l'Europe par les hommes, un facteur de mobilité et d'échanges, un «lieu» qui peut devenir cyberspace, pour expliciter les non-dits et faire tomber les appréhensions encore révélées par le récent passage de l'E.C.U. à l'EURO. Pour certains, E.C.U. ne signifiait pas seulement European Currency Unit. 

serait le premier, la stratégie de communication impulsée par la Commission, permettre de découvrir les "passerelles » entre les identités culturelles régionales ou nationales, hiérarchiser les positionnements, expérimenter les modes de construction d'une identité européenne qui « remonterait » par une stratégie de communication adaptée aux attentes et réalités. Ils constituent selon nous des vecteurs de communication privilégiés pour " appréhender les différences interculturelles en Europe..., les comprendre..., tisser et modéliser des liens durables dans les transactions humaines et commerciales" comme l'écrit M. Texier au terme d'une enquête dans les quinze pays, sur les «Styles de Management et Styles de Communication en Europe : Deux Dimensions Culturelles Proches ${ }^{14}$.

d'expérimenter les programmes européens ouverts par l'Union, voire l'intime conviction qu'ils sont concernés et pourraient en bénéficier. Ces programmes ne constituent pas moins une sorte de piste, d'archétype de communication pour résoudre le problème dialectique posé, « créer un espace intermédiaire... qui rende possible le dépassement des différences ». Ce mode s'inscrit dans le paradigme du compromis cher aux ergonomes, à F. Hubault notamment qui suppose une forme "d'espace public de discussion» et entrevoit après Y. Schwartz, "un dispositif à trois pôles: valeurs - savoirs -activités", fondements de l'identité elle-même, de la démarche qui nous occupe ici et s'illustrerait finalement avec l'Euro plutôt bien adapté en cette opportunité.

développer un mode de communication fondée sur la transaction, l'interaction, l'interrelation sur laquelle s'articulerait une communication institutionnelle respectueuse de cohérence, qui se garderait de toute manipulation, de déplacement $d u$ fond sur la forme et les relais, respectueuse d'un référentiel de l'information vraie autrement dit qui se prémunirait dès lors du procès d'intention, de " déstructuration de l'homme ${ }^{15}$ souvent fait aux mass médias.

En effet, pour reconnaitre les mérites de la communication selon la DG $\mathrm{X}$ avec ses réseaux et correspondants, ses interfaces entre organisation et identité, nous ne sommes pas moins d'accord avec Alain Touraine pour craindre «la séparation de l'information et des sujets qui la reçoivent... à cause d'un monde de flux, de réseaux et de signes qui détruit la possibilité de la communication... d'appropriation... (d')individuation ».

C'est pourquoi il convient de développer une communication qui donne à tous les citoyens-récepteurs européens la conviction qu'ils sont concernés, que l'on s'adresse à eux, qu'ils

ont un rôle ; autrement dit une communication de proximité vraie qui les incite à dresser comme un bilan sur le contenu, l'intérêt et la forme des messages diffusés, sur la relation, les signes discrets, l'usage possible pour eux... bref sur l'ensemble des éléments qui leur insufflerait le sentiment qu'il est répondu à leur inquiétudes présentes ou futures, à leurs attentes explicites ou implicites, en tant qu'habitants d'une région et d'un pays, participants d'une culture et d'une histoire; autrement dit une communication qui les atteigne dans leur vie quotidienne et les amène à remonter vers les grands concepts à partir d'un vécu reconnu authentique, à repérer les analogies et resituer leurs interrogations dans un contexte devenu compréhensible et intéressant, à se relier et se positionner dans le double espace.

Communication et organisation, 14 | 1998 


\section{Pour conclure}

41 En tant que symbole-image à double face de l'Europe et du pays, que symbole-reflet de la (non) réussite sociale intervenant dans les transactions et pérégrinations,l'Euro paraît bien le vecteur approprié à ce type de communication qui n'exclut pas la communication institutionnelle déjà mise en œuvre et indispensable pour relayer et amplifier.

Simplement la communication de proximité, interrelationnelle, renverse probablement l'ordre du processus, des domaines traités, des messages et leur mode d'apparition, puisqu'elle suit les besoins supputés d'un public-cible, privilégie les informations et modalités correspondantes, améliore la réceptivité et l'impact des documents écrits, sonores, visuels, repensés en conséquence, en même temps qu'elle suit les schémas de représentation et de cognition du récepteur, son cheminement identitaire et positionnel. Peut-être est-ce cela créer une société de communication, à rendre technologique ensuite, par adaptation de la démarche.

Peut-être est-il d'autres pistes. Celle-ci paraît convenir dans la mesure où elle ne récuse pas la stratégie de communication engagée mais la réintègre dans une perspective mieux appropriée nous semble-t-il, pour accompagner la nécessaire maturation des identités et éviter durant cette phase, les crises notamment souvent liées à l'apparition du sentiment d'insécurité ou de frustration. Plus que faire adhérer à un discours, l'objectif n'est-il pas d'insuffler la perception d'une identité européenne et la faire cohabiter avec une autre plus régionale ou nationale préexistante, partant, de relativiser et faire accepter la perception des territoires correspondants dans un rapport d'immanence? L'existence d'un espace-europe face au reste du monde devient alors la conséquence naturelle.

\section{BIBLIOGRAPHIE}

ABDALLAH-PRETCEILLE MV PORCHER L., Éducation et Communication Interculturelle, Paris, P.U.F., 1996.

ABDALLAH-PRETCEILlE M., THOMAS A., Relations et Apprentissage Interculturels, ouvrage publié avec le concours de l'Office Franco-Allemand pour la Jeunesse, Paris. A. Colin, 1995.

BALAGUE J., CANADELL I., Les Régions entre la Globalisation des Échanges et l'Union Européenne, Humanisme et Entreprise, 1995-214.

BELLON C, MiCNOT X., La Communication, Paris, Nathan, 1991.

BOURDIEU P., Questions de Sociologie, Paris, Éditions de Minuit, 1984.

BRETON P., PRoulX S., L'Explosion de la Communication, Paris/ Montréal, La Découverte/Boréal, 1989.

CAMilleri C, COHEN-EMERIQue M., Chocs de Cultures : Concepts et Enjeux, Pratiques de l'Interculturel, Paris, L'Harmattan, 1989.

CIALDINI R., Influence et Manipulations, Paris, First, 1991. 
DACHEUX E., Les O.N.G. sont-elles Victimes des Agences de Communication? Communication et Langages, $\mathrm{n}^{\circ} 102,1995$.

DACHEUX E., Les Composantes de l'Identité Culturelle de l'Union Européenne, Psychologie Europe, n³, vol. 3,1994.

DACHEUX E., Les Stratégies de Communication Persuasive dans L'Union Européenne, Paris, L'Harmattan, 1994. DEVAL Ph., Étude Comparative sur la Gestion des Conflits au Sein des Pays de l'Union Européenne; les Phases Conflictuelles et leur Mode de Régulation, Humanisme et Entreprise, 1996/220.

FISCHER-LOKOU J., Approche Psycho-Sociologique de la Procédure de Médiation dans le Cadre de la Négociation Formelle : Effet du pouvoir et de l'Innovation, Thèse de Doctorat, Université de Paris X sous la direction de S. Moscovici, 1994. GALLAND O, LECOMTE J, THÉVENOTL., « Les Valeurs de la jeunesse ", " Raisons de Vivre Raisons d'Agir ", "A l'Epreuve des Grands Principes ", Sciences Humaines $\mathrm{n}^{\circ}$ 79, Dossier Les Valeurs en Questions p. 19 à 33, Janvier 1998.

GERMAIN M., Histoire d'Entreprise et Recherche de Sens ; Restaurer la Mémoire et Exprimer l'Identité, Humanisme et Entreprise, 1996/217.

GIROUX N., La Communication Interne : une Définition en Évolution, Communication et Organisation n -5, 1er semestre 1994, Bordeaux, GREC/O.

GONDRAND F., Le langage des Eurocrates ou Comment Comprendre l'Europe dans Laquelle nous Sommes Embarqués, Humanisme et Entreprise, 1994-203.

Guillemot J. M., La Communication des Régions : Un Acte De Foi, Humanisme et Entreprise, 1996/215. HESS B., L'Entreprise face à l'Illetrisme, Paris, Anthropos, 1997.

HUBAULT F., Intervenir par l'Ergonomie : une Offre de Coopération/Coordination pour quelles Finalités? 3ème Congrès de la S.E.L.F., Bruxelles, 1996.

JULIEN C, La Communication Victime de Marchands ; Affairisme, Information et Culture de Masse, Paris, La Découverte, 1989.

LADMIRAL J.R., LIPIANSKY E.M., La Communication Interculturelle, Paris, Bibliothèque Européenne des Sciences de l'Éducation, A. Colin, 1991.

LEVY P., L'Intelligence Collective; Pour une Anthropologie du Cyberspace, Paris, La Découverte, Sciences et Société, 1995.

LEYENS J.P., Psychologie Sociale, Liège, Mardaga, 1979.

MARCHETEAU M., L'Europe : Plurilinguisme ou Confusion des Langues? Peut-on construire Babel ?

Humanisme et Entreprise, 1994-204.

MARTIN M., Communication et Médias de Masse ; Culture, Domination et Opposition, Québec, Presses de l'Université du Québec, 1991.

mattelart A., La Communication-Monde, Histoire des Idées et des Stratégies, Paris, La Découverte, 1992.

MIÈGE B., Médias et Communication en Europe, Grenoble, Presses Universitaires de Grenoble, 1990. MONETTE P.Y., L'Europe, État d'Urgence, Paris, Desclée de Brouwer, 1997.

MORTN E., Penser l'Europe, Paris, Gallimard, 1987.

MUCCHIELLI A., L'Identité, Que sais-je, Paris, P.U.F., 3º́dition corrigée, 1994.

MUCCHœLli A., Psychologie de la Communication, Paris, P.U.F., 1995. 
QURNTON P., Le Design Graphique, Interface des Changements, Humanisme et Entreprise, 1996, 218.

REUCHLIN M., Introduction à la Recherche en Psychologie, Paris, Nathan, 1992.

SAJNSAUlteu R, La Construction des Identités au Travail, Sciences Humaines, Hors série n 20, p. 40 à

43, Mars-Avril 1998.

Sciences de la Société, Territoires Frontaliers-Discontinuité et Cohésion, $n^{\circ} 37$, Toulouse, Presses Universitaires du Mirail, $19 \%$.

Sciences de la Société, Les Régions dans l'Europe, n 34, Toulouse, Presses Universitaires du Mirail, 1995.

TEXIER M., Styles de Management et Styles de Communication en Europe : Deux Dimensions Culturelles Proches? Humanisme et Entreprise, 1994/205.

TOLLIS E, La Parole et le Sens; le Guillaumisme et l'Approche contemporaine du langage, Paris, A. Colin, 1994. VAlence G., La Légende du Franc, de 1360 à Demain, Paris, Flammarion, 1996.

watzlawick P., La Réalité de la Réalité, Paris, Edition du Seuil, 1984.

Documents DG X :

DE DEUS PDMHEIRO ]., Information, Communication, Transparence, 1994.

O REJA M., Mieux Informer les Citoyens de l'Union. Une stratégie Globale, 1995.

DG XIII de la Commission Européenne, Les Régions en Marche vers la Société de l'Information, 1996.

Rapport d'Étude Quantitative, Les Réactions des Particuliers à l'Egard de la Monnaie Unique Européenne, C.E.R.P., 1997.

\section{NOTES}

1. Parce qu'elle nous semble particulièrement adaptée a ce que vise la DG X nous nous référons fondamentalement a la définition de l'Identité donnée par A. Mucchielli à la page 5 de « son » Que sens-je? " ensemble de critères de définition d'un sujet et sentiment interne Ce sentiment d'identité est composé de différents sentiments : sentiment d'unité, de cohérence, d'appartenance, de valeur, d'autonomie et de confiance organisés autour d'une volonté d'existence ».

2. Comment fonctionne l'Union Européenne?

- L'Union Européenne, quel intérêt pour moi?

- Comment l'union Européenne protège-t-elle notre environnement?

- Comment l'Union Européenne gère-t-elle l'Agriculture et la Pèche?

- Quels sont mes droits en tant que consommateur?

- Education et Formation, Deux armes contre le chômage : qui peut participer ? où et comment postuler?

- Résider dans un autre pays de l'Union Européenne : Comment faire reconnaître vos droits et vous en prévaloir?

3. Quinton, «Le Design Graphique, Interface des changements ». Humanisme et Entreprise, 1996/218, p. 82

4. P. Levy, «L'Intelligence collective » : Paris, La Découverte, 1995, p. 21.

5. P. Levy, Ibid

6. G A. Hillery cité par E. Dacheux, «Les Stratégies de Communication persuasive dans l'Union Européenne», p. 68.

7. C. Camilleri, «Chocs de Cultures», Paris, l'Harmattan, 1989, p. 27. 
8. R. Cialdini, «Influence et Manipulations » Paris, First, 1991, p. 116.

9. J.R. Ladmiral, "La Communication Interculturelle», Paris, La Bibliothèque Européenne des Sciences de l'Éducation, A. Colin, 1991, p. 11.

10. Ibid., p. 21

11. M. Abdallah Pretceille, "Pédagogie Interculturelle: Bilan et Perspectives », l'Interculturel en Éducation et en Sciences Humaines, p. 31.

12. E. Dacheux. op.cit., p. 43

13. A. Mucchielli,op.cit., p. 91.

14. Humanisme et Entreprise, 1994/ 205, p. 102.

15. A. Mucchielli, op.cit., p. 99.

\section{RÉSUMÉS}

Pour exister, l'Union Européenne doit instiller la notion de territoire indissociable de l'identité. Seule une communication de proximité vraie peut favoriser la nécessaire maturation de celle-ci au travers d'un nécessaire relationnel sans déstructurer ni induire un reniement du culturel originel. En effet, seule une communication de proximité vraie, cependant orchestrée par l'Institution, peut intégrer les facteurs promotionnels et fédérateurs de la communication corporate, tout en donnant aux citoyens européens le sentiment qu'elle est développée pour eux et qu'il est répondu à leurs inquiétudes ou attentes, dans la mesure où elle les atteint dans leur quotidien, leur permet de se resituer dans un contexte redevenu compréhensible, exclut en fait « la séparation de l'information et des sujets qui la reçoivent ».

To insure its own existence, the European Union must endeavour to promote the union of territory which is intimately linked with the notion of identity. Close-range communication, orchestrated by the institution itself is the only tape of communication able to integrate the corporate communication federating elements while convincing the citizens that the whole process is aimed at them and at their expectations, in as much as it purtains to their daily life and helps them to reframe their own situation.

\section{INDEX}

Mots-clés : identité, espace, culture(s), proximité, cohérence

\section{AUTEUR}

\section{JEAN-PIERRE CALLÉGARI}

Jean-Pierre Callegari est Maître de conférences à l'Université de Valenciennes et du Hainaut Cambrésis. Ses recherches portent sur la communication des entreprises et la qualité. 